\title{
A narrative review of proteolytic targeting chimeras (PROTACs): future perspective for prostate cancer therapy
}

\author{
Xuanrong Chen ${ }^{1 \#}$, Haishan Shen ${ }^{2 \#}$, Yi Shao ${ }^{1 \#}$, Qianwang $\mathrm{Ma}^{1}$, Yuanjie Niu ${ }^{1}$, Zhiqun Shang ${ }^{1}$ \\ ${ }^{1}$ Department of Urology, Tianjin Institute of Urology, The Second Hospital of Tianjin Medical University, Tianjin, China; ${ }^{2}$ Urology Development, \\ Chu Hsien-I Memorial Hospital \& Tianjin Institute of Endocrinology, Tianjin Medical University, Tianjin, China \\ Contributions: (I) Conception and design: X Chen, Y Niu, Z Shang; (II) Administrative support: Q Ma; (III) Provision of study materials or patients: \\ X Chen, H Shen, Y Shao; (IV) Collection and assembly of data: X Chen, H Shen, Y Shao, Q Ma; (V) Data analysis and interpretation: X Chen, H \\ Shen, Y Shao; (VI) Manuscript writing: All authors; (VII) Final approval of manuscript: All authors. \\ \#These authors contributed equally to this work. \\ Correspondence to: Prof. Zhiqun Shang. Department of Urology, Tianjin Institute of Urology, the Second Hospital of Tianjin Medical University, \\ Tianjin, China. Email: zhiqun_shang@tmu.edu.cn.
}

\begin{abstract}
Proteolysis-TArgeting Chimeras (PROTACs) technology, as a strategy to chemically knock down transcription factors at the protein levels, can hijack the ubiquitin-proteasome degradation system to initiate the intracellular ubiquitin-proteasome hydrolysis process to degrade proteins. In the past, the development of drugs that target transcription factors has been greatly restricted, and even historically transcription factors have been regarded as "undruggable targets". PROTAC technology breaks through this limitation with its unique targeting design. With several generations of technical innovation, PROTACs have become more mature and continue to make breakthroughs in the field of targeted therapy including prostate cancer (PCa), with a new strategy for the development of anti-tumor targeted drugs. PROTACs have all the advantages of existing small molecule inhibitors, are easy to administer orally, have good cell permeability, and have wider targeting profiles compared to conventional inhibitors. The disadvantage of PROTACs is the noncancer specificity, off-target and sustained-release control, due to its catalytic role. Some androgen receptor (AR) and CDK4/6 degraders have advanced the field of PCa treatment, which is being further modified given the effects of these degraders in preclinical and clinical studies. This review summarizes in detail the technological progress and challenges that have been faced with PROTACs, the progress of research on $\mathrm{PCa}$, and the prospective future of PROTACs development.
\end{abstract}

Keywords: Proteolytic targeting chimera; prostate cancer (PCa); transcription factor

Submitted Oct 21, 2020. Accepted for publication Dec 31, 2020.

doi: $10.21037 /$ tau-20-1357

View this article at: http://dx.doi.org/10.21037/tau-20-1357

\section{Introduction}

Transcription factors are a class of proteins that can bind to specific DNA sequences and are known to be highly active in humans. They can form complexes either alone or with other proteins, and play a pivotal role in regulating specific gene expression (1).
In tumors, a variety of direct or indirect mechanisms deregulate both the activity and content of transcription factors, and dysregulation of transcription factors has also become a hallmark characteristic of the tumor $(2,3)$. Changes in chromatin structure, amplification/deletion of genes encoding the transcription factors, and mutations

^ ORCID: 0000-0003-4694-4579. 
directly affect the activity of transcription factors; changes in non-coding sites, transcription factors and abnormal distribution may also indirectly affect the activity of transcription factors. However, targeting chemotherapeutic strategies has been greatly restricted, and even historically, transcription factors have been regarded as "undruggable targets". Unlike kinase proteins, kinases often have intracellular active sites that are easier to predict and recognize. Transcription factors usually work through protein-DNA or protein-protein interactions. For proteinDNA interactions, the DNA binding interface offers a highly positive charge and a convex structure, which is not conducive to target. For protein-protein interactions, the surface of the binding interface is usually flat, and the absence of a pocket structure such as a kinase active site also makes this drug development a great challenge $(4,5)$. Despite these challenges, several generations of scientists have developed several methods to target transcription factors, including RNA interfering (RNAi), targeting of post-translational modification and degrading transcription factors with PROTACs, and targeting of intrinsically disordered regions of transcription factors and targeting the auto-inhibited state of transcription factors (6-10). The development of these new targeted drugs will greatly advance the treatment of future tumors and offer hope to patients.

In this review, we review the current knowledge regarding the PROTAC technology, the research process and the agents currently available for the treatment of $\mathrm{PCa}$, and future directions for the development of the PROTAC technology. A comprehensive literature search was conducted in the PubMed/Medline, Cochrane, Scopus and ClinicalTrials databases using the keywords "prostate cancer" OR "prostate carcinoma" OR "castration-resistant prostate cancer" AND "PROTACs" OR "proteolytic targeting chimera" OR "PROTAC". We chose to include the most relevant reports based on the quality, applicability, and development of the research. We present the following article in accordance with the Narrative Review reporting checklist (available at http://dx.doi.org/10.21037/tau-201357).

\section{Background}

PROTAC (PROteolysis-TArgeting Chimera, PROTAC) is a special proteolytic targeted chimera technology (11).
PROTAC is a strategy to chemically knockdown transcription factors at the protein level. One end of the chimera contains a specific binding ligand for protein degradation, and the other end is a binding ligand for the E3 ubiquitin ligase, with both ends being connected by a linker (12).

Ubiquitin-mediated protein degradation is a critical pathway by which cells regulate their cellular protein levels. The ubiquitin-mediated protein degradation pathway can degrade more than $80 \%$ of the ubiquitous proteins in cells. This pathway plays a role in almost all cell life processes including cell cycle regulation, cell proliferation, cell apoptosis, and signaling pathways within and outside the cell (13). Among these enzymes, the E1 ubiquitin activating enzyme, E2 ubiquitin conjugating enzyme and E3 ubiquitin ligase co-operate with each other to label the substrate protein for ubiquitination, and then recruit the proteasome for degradation (14). The E3 ubiquitin ligase has clear specificity for the labeling process of substrate proteins, but also provides a theoretical and applied basis for the targeted labeling degradation of PROTACs. The special chimera structure of PROTACs narrows the space between the target protein and the intracellular E3 ubiquitin ligase by self-folding to form a target protein-PROTACs chimera-E3 ubiquitin ligase terpolymer, which makes the E3 ubiquitin ligase ubiquitinate the target protein, and then uses the ubiquitin-proteasome degradation pathway to initiate the intracellular ubiquitinating hydrolysis process to complete the process of chemically targeted degradation of the protein (15).

\section{Research progress of proteolytic targeted chimera technology}

In 2001, the research group of Professors Deshaies and Crews first reported the chemical degradation of proteins (11). The intracellular ubiquitination and degradation of specific proteins was subsequently significantly expanded and utilized. As one of the first generation of PROTACs, PROTAC-1 is one of the first PROTAC molecules. This is the first generation of peptidebased PROTACs technology, which relies on the application of comprehensive analysis of substrate binding sites and structural information for the identification of the proteins. One end of the PROTAC- 1 molecule utilizes ovalicin (OVA) to cooperate with the histidine at the 231st active site of the 
amino peptidase-2 (MetAP-2) peptide. The other end of the phospho-peptide of the nuclear factor $\kappa \mathrm{B}$ inhibitor $\alpha(\mathrm{NF}-\kappa \mathrm{B}$ inhibitor $\alpha, \mathrm{I} \kappa \mathrm{B} \alpha$ ) structure can bind to $\beta$-TRCP in the E3 ubiquitin ligase protein complex. PROTAC-1 binds the MetAP-2 protein and $\beta$-TRCP complex tightly together, activating the ubiquitin-proteasome degradation pathway to degrade the MetAP-2 protein. Several subsequent peptide-based PROTACs, such as PROTAC-2, which uses the androgen receptor (AR) ligand dihydrotestosterone to link it with the I $\kappa \mathrm{B} \alpha$ phospho-peptide to degrade the AR. PROTAC- 3 uses the estrogen receptor ligand estradiol and $\mathrm{I} \kappa \mathrm{B} \alpha$ phospho-peptide to degrade the estrogen receptor (16). In addition, the larger molecular weight of the E3 ubiquitin ligand results in unstable structure and poor cell permeability. Scientists continue to discover and synthesize small molecule ligands for E3 ligases and their corresponding derivative structures, such as CRBN, cIAP, VHL and other specific E3 ligase small molecule ligands (17-20). In view of the fact that the first-generation PROTACs needs to be highly dependent on the complex use of the target protein substrate site and structure information, the second generation PROTACs based on small molecule has designed. This improvement allows PROTACs to achieve targeted degradation of common oncogenic genes and transcription factors in a variety of tumors, such as the BCR-ABL fusion protein in chronic myeloid leukemia, and the BRD4 protein in acute myeloid leukemia and lymphoma, the estrogen receptor protein in breast cancer, the TACC 3 protein in fibrosarcoma, etc. (21-23).

Researchers have also found the problems of off-target and sustained-release control of tissue distribution of these small molecule PROTACs. In response to this, a research group developed the third generation PROTACs in 2013. This generation of PROTACs can specifically control the temporal and spatial distribution of PROTACs in the tissues to better exert their efficacy, and to reduce offtarget distribution in the tissues. PhosphoPROTACs, known as phosphate-dependent PROTACs, use activated phosphokinase as a target signal to achieve controllable degradation (24). There are also PROTACs that use ultraviolet light sources in photodynamic therapy to achieve controllable PROTACs that can be light-controlled on and off (25).

The third-generation controllable PROTACs have become a spot for drug research. PROTACs are typically a chimera in molecular structure, with the linker connecting the two ends of the structure. The choice of a linker, the length of the linker, and the binding site between the linker and the molecules at both ends also have a greater impact on the activity of PROTACs (26). Taking estrogen receptortargeted PROTACs as an example, when the linker causes a certain gap between the target protein binding site and the E3 ubiquitin ligase, PROTACs with 16 atom chains of the linker have the highest degradation effects (27). Drugs with PROTACs as the universal design principle are becoming increasingly common, and it is believed that controllable drugs for PROTACs will become a reality in the coming decades $(28,29)$.

\section{Advantages and disadvantages of PROTACs}

PROTAC has gained many advantages since its inception, particularly in its unique targeting and chemical degradation. The site-directed targeting of PROTACs does not rely on directly inhibiting the active site of the kinase or inhibiting the interaction site for the purpose of inhibiting protein function (30). When designing targeted sites, there is no need to specifically analyze the active site and interaction site of the protein, nor do we consider the spatial folding structure of the site as a whole. In theory, as long as a functional site is not involved, this approach can be used to design PROTACs.

The chemical degradation of PROTACs involves the cell's own ubiquitin-proteasome protein degradation pathway. This degradation causes the protein to "disappear" within the cell after ubiquitination and hydrolysis, as opposed to specifically targeting small molecule compounds that block the active sites. This chemical degradation can minimize the shortcomings of the short half-life of small molecule compounds, and a smaller dose of PROTACs can produce significant degradation effects. Since the protein has to be re-synthesized after targeted degradation to restore its distribution and function, the effect of this chemical degradation should be more durable and efficient than small-molecule compounds that block its active site. Winter et al. compared the therapeutic effects of BRD4 degraders with that of BRD4 inhibitors alone. By using BRD4 inhibitors alone, BRD4 degraders can more efficiently and selectively induce protein degradation in vitro and in vivo, as well as delay the progression of leukemia in mice (31). With the continual discovery of small molecule 
ligands for E3 ubiquitin ligase, the range of possibilities for the development of PROTACs has been infinitely broadened (32).

However, PROTACs are imperfect, and many potential problems have been discovered in clinical applications. Some PROTACs have poor pharmacokinetics due to their large molecular weight and cannot be easily absorbed by oral administration. The latter small molecule PROTACs improved this shortcoming and improved the oral bioavailability. Although several E3 ubiquitin ligases have been discovered, the types of small molecule ligands that can be properly matched and designed are still limited. The functioning of PROTACs is heavily dependent on the functional E3 ubiquitin ligase and the ubiquitin-proteasome protein degradation system. Once the degradation pathway fails or the E3 ubiquitin ligase mutates, drug adaptation or acquired resistance may occur. Therefore, predictive biomarkers need to be incorporated into the clinical treatment practice of PROTACs to address these issues (33).

The existing small-molecule PROTACs should have a small molecular weight after improvement, and they can enter and exit a large number of tissue cells relatively freely in the human body, resulting in a wide tissue distribution, causing serious toxic side effects. The third-generation PROTACs are a more practical strategy, but their application is limited due to the potential DNA damage and poor penetration of ultraviolet rays such as UVA. Therefore, it is possible to consider developing other light sources with better penetrability to precisely control the release of PROTACs to expand the scope of applications and reduce side effects (34).

\section{Application of proteolytic targeting chimera technology in prostate cancer}

In $\mathrm{PCa}$, the androgen receptor (AR) is a key protein molecule and transcription factor closely related to tumorigenesis and development, and is also a drug target for various treatment methods including androgen-deprivation therapy $(35,36)$. Unfortunately, most patients receiving endocrine therapy will enter the castration-resistant prostate cancer (CRPC) stage within one to two years, progress to a difficult malignant stage and eventually die from the disease $(37,38)$. For CRPC patients, the advent of $\mathrm{AR}$ antagonist drugs such as enzalutamide has a significant clinical benefit for these patients (39-41). However, even after receiving these next-generation $\mathrm{AR}$ antagonist drugs, patients continue to become resistant and incurable (42-44). This suggests that the still-active AR signaling pathway urgently needs to be settled.

As early as 2015, Professor Crews designed a selective androgen receptor degrader (SARD; no.SARD279). This small molecule contains a hydrophobic group linked to an AR ligand, which can induce degradation of AR proteins, reduce the expression of $\mathrm{AR}$ target genes and inhibit the proliferation of androgen-dependent PCa cells (45). This innovative degradation common can overcome certain resistance mechanisms that are common to traditional drugs. In 2018, Professor Crews' research group continued to expand on this technique by combining it with PROTAC technology to design a more efficient AR degradation agent, named ARCC-4 (46). ARCC-4 is a low-molecularweight AR degradation agent that can degrade about $95 \%$ of the AR protein in PCa cells. It can significantly inhibit the proliferation of prostate tumor cells ( $\mathrm{VCaP}, \mathrm{LNCaP}$, $\mathrm{PC} 3$, etc.), and also degrade some clinical -related AR point mutants (AR-T877A, AR-H874Y, AR-F876L, AR-L702H, AR-M896V and other point mutants). Unlike enzalutamide, ARCC-4 also has a significant anti-proliferative effect under high androgen concentration conditions, which suggests that using this degradation technology to overcome enzalutamide resistance may be a breakthrough treatment for these patients.

At the same time, a research group led by Professor Wang from the University of Michigan designed a highly effective AR-targeted PROTAC degradation agent (ARD-69) (47). In vitro experiments have shown that $\mathrm{ARD}-69$ induces $\mathrm{AR}$ protein degradation in AR-positive PCa cell lines (LNCaP, $\mathrm{VCaP}$ and 22Rv1) in a dose- and time-dependent manner. At the same time, ARD-69 also reduces the endogenous AR protein in these PCa cell lines to below 95\%, and effectively inhibits the expression of downstream regulatory genes in the AR signaling pathway. ARD-69 can effectively inhibit cell growth and has an effect that is more than 100 times that of the AR antagonist enzalutamide. In vivo experiments have shown that a single dose of ARD-69 can effectively reduce the levels of AR protein in mouse xenograft tumor tissue. These data suggest that AR-targeted degradation therapy based on PROTACs may become a more viable option for AR-positive CRPC patients. Professor Chinnaiyan's research group has also developed a new AR degrader (AR PROTAC degrader, no. ARD-61) based on 
Table 1 The basic chemical structures, biological activities, physiochemical properties of the compounds. AR, androgen receptor; VHL, Von Hippel-Lindau; CDK4/6, cyclin dependent kinase 4/6

\begin{tabular}{llllll}
\hline Name & Target & E3 ligase & Molecular weight $(\mathrm{g} / \mathrm{mol})$ & Molecular formula & Degradation activity \\
\hline ARCC-4 & AR & VHL & $1,024.2$ & C53H56F3N7O7S2 & $\begin{array}{l}\text { Degradation of AR protein in VCaP cells } \\
\text { after 20 } \mathrm{h} \text { treatment }\end{array}$ \\
ARD69 & AR & VHL & $1,129.8$ & C62H74CIFN8O7S & $\begin{array}{l}\text { Degradation of AR protein in LNCaP/ } \\
\text { VCaP/22Rv1 cells after 24 } \mathrm{h} \text { treatment }\end{array}$ \\
ARD61 & AR & VHL & $1,095.8$ & C61H71CIN8O7S & $\begin{array}{l}\text { Degradation of AR protein in LNCaP/VCaP } \\
\text { cells after } 6 \mathrm{~h} \text { treatment }\end{array}$ \\
CST620 & CDK4/6 & VHL & $1,064.5$ & C55H73N11O9S & $\begin{array}{l}\text { Degradation of CDK4/6 in several leukemia, } \\
\text { myeloma and breast cancer cell lines }\end{array}$ \\
\hline
\end{tabular}

PROTACs and has carefully elucidated its mechanism of action. ARD-61 overcomes the existing resistance process to anti-hormone therapies by directly depleting the AR protein in both PCa and breast cancer cell lines with AR-positive features (48-50). Through in vivo and in vitro experiments, studies have found that compared to enzalutamide, ARD61 has stronger anti-proliferation and pro-apoptosis effects, and significantly inhibits the regulation of the AR signaling in cancer cells, while still exerting an inhibitory effect in the enzalutamide resistance model. Further analysis found that although ARD-61 cannot bind and target AR-V7 (AR splice variant 7), it can still inhibit the growth of tumor cells with AR-V7 overexpression. Studies have shown that AR is still the main driving factor for PCa, and new AR degraders have a wider clinical significance for patients who are resistant to AR antagonists. The Arvinas company has been approved by the US Food and Drug Administration in 2019 to initiate a phase I clinical trial (NCT03888612) of oral PROTACs (ARV-110) targeting the AR protein in patients with metastatic CRPC. Another oral PROTAC (ARV-471) targeting estrogen receptor protein has also been approved for the Phase I clinical trial (NCT04072952) in patients with ER positive/HER2 negative locally advanced or metastatic breast cancer.

The mammalian cyclin-dependent kinases (CDKs) contain a cell cycle related sub-family (CDK1, CDK2, CDK4, CDK6) (51,52). In clinics, CDK4/6 inhibitors have emerged as a powerful class of agents for estrogen receptor positive breast cancer treatment (53). Targeting the cell cycle represents a core attack on a defining feature of cancer, given the effects of AR signaling on the cell cycle in PCa. This is a key component of the treatment of cancer. Given the importance of CDK4 and CDK6, we propose that CDK4/6 inhibitors and novel strategic combinatorial therapies have the potential to improve patients' overall survival and quality of life (54). Steinebach et al. designed a VHL-based PROTAC (CST620) exhibiting dual activity against CDK4 and CDK6, and showed potent and longlasting degrading activity in human and mouse cells and inhibited proliferation of several leukemia, myeloma and breast cancer cell lines (55). This attractive approach for targeted degradation of CDK4/6 may be further tested in PCa.

In summary, the beginning of clinical trials of oral PROTAC targeting transcription factors ends the period in which transcription factors are "undrugable". The development of these novel and exciting clinical trials provides great opportunities for future clinical applications. The basic properties of the compounds described above are listed in Table 1 and are depicted in Figure 1.

\section{Future directions}

PROTACs have almost all the advantages of existing small molecule inhibitors, are easy to administer orally, have good cell permeability and solubility, and have extensive targeting profiles. As a transcription factor, the $\mathrm{AR}$ protein in $\mathrm{PCa}$ only needs to be targeted by PROTACs and does not need to consider whether it can block its active site or interfere with its interaction with other proteins, making it easier to develop and synthesize $(56,57)$. With the progression of $\mathrm{PCa}$, accumulating studies have found that the AR signaling pathway is inactivated in highly malignant neuroendocrine prostate cancer, which suggests that we need to develop appropriate drugs for patients with neuroendocrine prostate cancer. PROTACs are believed to be a progressive solution to this problem and have become an important tool in cancer treatment (58). 
Translational Andrology and Urology, Vol 10, No 2 February 2021

959

A

ARCC4

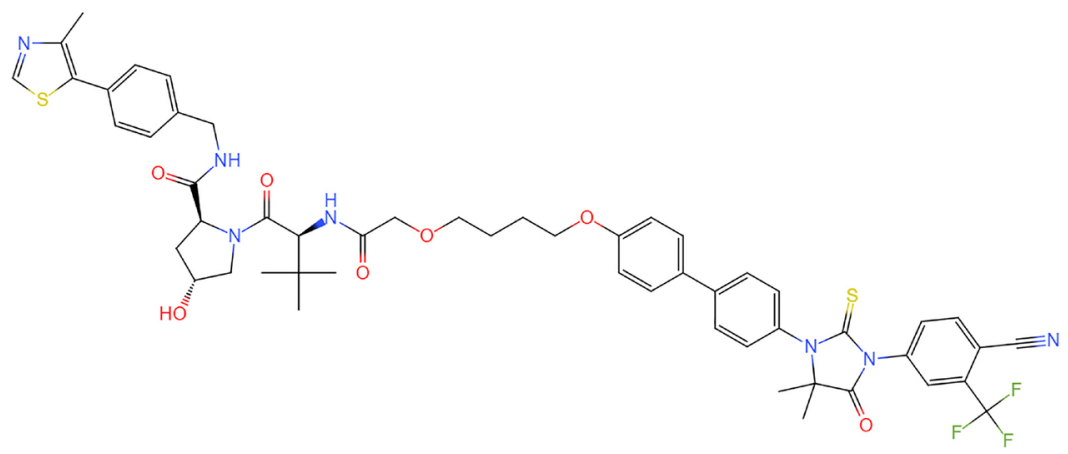

B

ARD 69

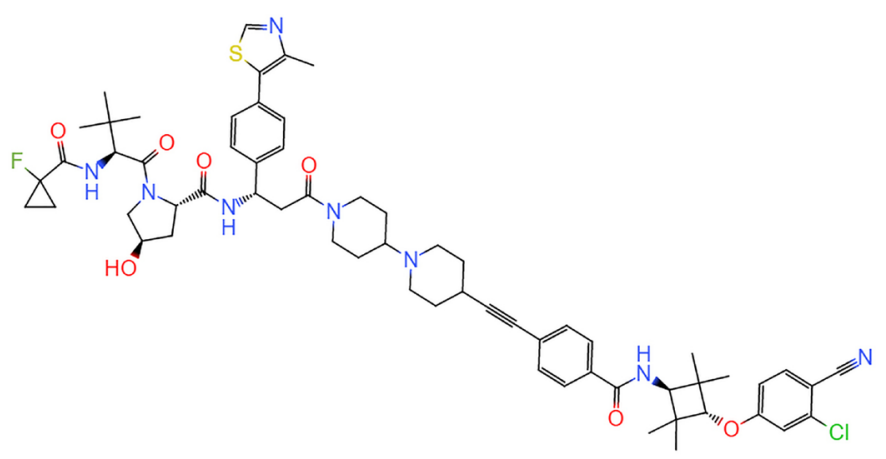

C

ARD61

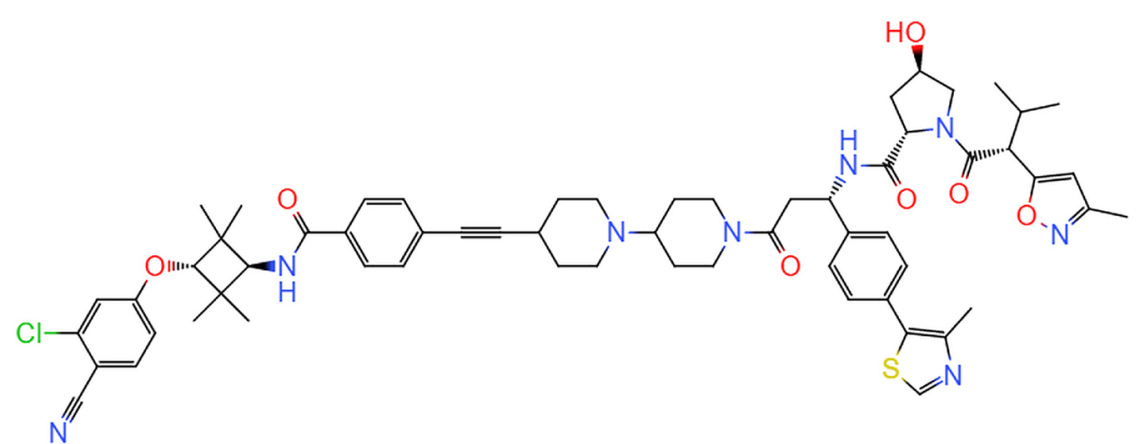

D<smiles>C/C=C(\C=C/Nc1scnc1C)[C@H](C)NN[C@H]1CC(O)CN1C(=O)[C@H](NC(=O)COCCOCCOCCN1CCN(c2ccc(Nc3ncc4c(C)c(C(C)=O)c(=O)n(C5CCCC5)c4n3)nc2)CC1)C(C)(C)C</smiles>

Figure 1 Chemical structures of the reported degraders. (A) Chemical structures of ARCC-4. (B) Chemical structures of ARD 69. (C) Chemical structures of ARD61. (D) Chemical structures of CST60.

(c) Translational Andrology and Urology. All rights reserved.

Transl Androl Ural 2021;10(2):954-962 | http://dx.doi.org/10.21037/tau-20-1357 


\section{Acknowledgments}

Funding: This work was supported by National Natural Science Foundation of China (grand No. 82072851, 81872100 and 81772756) and Natural Science Foundation of Tianjin (18PTLCSY00030 and 19JCYBJC24900).

\section{Footnote}

Reporting Checklist: The authors have completed the Narrative Review reporting checklist. Available at http:// dx.doi.org/10.21037/tau-20-1357

Peer Review File: Available at http://dx.doi.org/10.21037/ tau-20-1357

Conflicts of Interest: All authors have completed the ICMJE uniform disclosure form (available at http://dx.doi. org/10.21037/tau-20-1357). Dr. YN serves as an unpaid editorial board member of Translational Andrology and Urology from Mar 2015 to Feb 2021. The other authors have no conflicts of interest to declare.

Ethical Statement: The authors are accountable for all aspects of the work in ensuring that questions related to the accuracy or integrity of any part of the work are appropriately investigated and resolved.

Open Access Statement: This is an Open Access article distributed in accordance with the Creative Commons Attribution-NonCommercial-NoDerivs 4.0 International License (CC BY-NC-ND 4.0), which permits the noncommercial replication and distribution of the article with the strict proviso that no changes or edits are made and the original work is properly cited (including links to both the formal publication through the relevant DOI and the license). See: https://creativecommons.org/licenses/by-nc-nd/4.0/.

\section{References}

1. Lee TI, Young RA. Transcriptional regulation and its misregulation in disease. Cell 2013;152:1237-51.

2. Lambert M, Jambon S, Depauw S, et al. Targeting Transcription Factors for Cancer Treatment. Molecules (Basel, Switzerland) 2018;23:1479.

3. Rajagopal C, Lankadasari MB, Aranjani JM, et al. Targeting oncogenic transcription factors by polyphenols: A novel approach for cancer therapy. Pharmacol Res
2018;130:273-91.

4. Arkin MR, Tang Y, Wells JA. Small-molecule inhibitors of protein-protein interactions: progressing toward the reality. Chem Biol 2014;21:1102-14.

5. Ran X, Gestwicki JE. Inhibitors of protein-protein interactions (PPIs): an analysis of scaffold choices and buried surface area. Curr Opin Chem Biol 2018;44:75-86.

6. Pufall MA, Graves BJ. Autoinhibitory domains: modular effectors of cellular regulation. Annu Rev Cell Dev Biol 2002;18:421-62.

7. Chen YNP, LaMarche MJ, Chan HM, et al. Allosteric inhibition of SHP2 phosphatase inhibits cancers driven by receptor tyrosine kinases. Nature 2016;535:148-52.

8. Zlatev I, Castoreno A, Brown CR, et al. Reversal of siRNA-mediated gene silencing in vivo. Nat Biotechnol 2018;36:509-11.

9. Zhou J, Oldfield CJ, Yan W, et al. Intrinsically disordered domains: Sequence $\rightarrow$ disorder $\rightarrow$ function relationships. Protein Sci 2019;28:1652-63.

10. Liu J, Ma J, Liu Y, et al. PROTACs: A novel strategy for cancer therapy. Semin Cancer Biol 2020;67:171-9.

11. Sakamoto KM, Kim KB, Kumagai A, et al. Protacs: chimeric molecules that target proteins to the Skp1Cullin-F box complex for ubiquitination and degradation. Proc Natl Acad Sci U S A 2001;98:8554-9.

12. Wang $Y$, Jiang $X$, Feng F, et al. Degradation of proteins by PROTACs and other strategies. Acta Pharm Sin B 2020;10:207-38.

13. Deng L, Meng T, Chen L, et al. The role of ubiquitination in tumorigenesis and targeted drug discovery. Signal Transduct Target Ther 2020;5:11.

14. Zheng N, Shabek N. Ubiquitin Ligases: Structure, Function, and Regulation. Annu Rev Biochem 2017;86:129-57.

15. Neklesa TK, Winkler JD, Crews CM. Targeted protein degradation by PROTACs. Pharmacol Ther 2017;174:138-44.

16. Sakamoto KM, Kim KB, Verma R, et al. Development of Protacs to target cancer-promoting proteins for ubiquitination and degradation. Mol Cell Proteomics 2003;2:1350-8.

17. Buckley DL, Van Molle I, Gareiss PC, et al. Targeting the von Hippel-Lindau E3 ubiquitin ligase using small molecules to disrupt the VHL/HIF-1 $\alpha$ interaction. J Am Chem Soc 2012;134:4465-8.

18. Sekine K, Takubo K, Kikuchi R, et al. Small molecules destabilize cIAP1 by activating auto-ubiquitylation. J Biol Chem 2008;283:8961-8. 
19. Krönke J, Fink EC, Hollenbach PW, et al. Lenalidomide induces ubiquitination and degradation of CK1 $\alpha$ in $\operatorname{del}(5 q)$ MDS. Nature 2015;523:183-8.

20. Matyskiela ME, Lu G, Ito T, et al. A novel cereblon modulator recruits GSPT1 to the CRL4(CRBN) ubiquitin ligase. Nature 2016;535:252-7.

21. Okuhira K, Demizu Y, Hattori T, et al. Development of hybrid small molecules that induce degradation of estrogen receptor-alpha and necrotic cell death in breast cancer cells. Cancer Sci 2013;104:1492-8.

22. Ohoka N, Nagai K, Hattori T, et al. Cancer cell death induced by novel small molecules degrading the TACC3 protein via the ubiquitin-proteasome pathway. Cell Death Dis 2014;5:e1513.

23. Zengerle M, Chan KH, Ciulli A. Selective Small Molecule Induced Degradation of the BET Bromodomain Protein BRD4. ACS Chem Biol 2015;10:1770-7.

24. Hines J, Gough JD, Corson TW, et al. Posttranslational protein knockdown coupled to receptor tyrosine kinase activation with phosphoPROTACs. Proc Natl Acad Sci U S A 2013;110:8942-7.

25. Xue G, Wang K, Zhou D, et al. Light-Induced Protein Degradation with Photocaged PROTACs. J Am Chem Soc 2019;141:18370-4.

26. Lu J, Qian Y, Altieri M, et al. Hijacking the E3 Ubiquitin Ligase Cereblon to Efficiently Target BRD4. Chem Biol 2015;22:755-63.

27. Cyrus K, Wehenkel M, Choi EY, et al. Jostling for position: optimizing linker location in the design of estrogen receptor-targeting PROTACs. ChemMedChem 2010;5:979-85.

28. Buckley DL, Raina K, Darricarrere N, et al. HaloPROTACS: Use of Small Molecule PROTACs to Induce Degradation of HaloTag Fusion Proteins. ACS Chem Biol 2015;10:1831-7.

29. Scudellari M. Protein-slaying drugs could be the next blockbuster therapies. Nature 2019;567:298-300.

30. Xia L, Liu W, Song Y, et al. The Present and Future of Novel Protein Degradation Technology. Curr Top Med Chem 2019;19:1784-8.

31. Winter GE, Buckley DL, Paulk J, et al. DRUG DEVELOPMENT. Phthalimide conjugation as a strategy for in vivo target protein degradation. Science 2015;348:1376-81.

32. Schapira M, Calabrese MF, Bullock AN, et al. Targeted protein degradation: expanding the toolbox. Nat Rev Drug Discov 2019; 18:949-63.

33. Ottis P, Palladino C, Thienger P, et al. Cellular Resistance
Mechanisms to Targeted Protein Degradation Converge Toward Impairment of the Engaged Ubiquitin Transfer Pathway. Science 2018;359:679-84.

34. Chen S, Weitemier AZ, Zeng X, et al. Near-infrared deep brain stimulation via upconversion nanoparticle-mediated optogenetics. Science (New York, NY) 2018;359:679-84.

35. Culig Z, Santer FR. Androgen receptor signaling in prostate cancer. Cancer Metastasis Rev 2014;33:413-27.

36. Pagliarulo V, Bracarda S, Eisenberger MA, et al. Contemporary role of androgen deprivation therapy for prostate cancer. Eur Urol 2012;61:11-25.

37. Grasso CS, Wu YM, Robinson DR, et al. The mutational landscape of lethal castration-resistant prostate cancer. Nature 2012;487:239-43.

38. Kirby M, Hirst C, Crawford ED. Characterising the castration-resistant prostate cancer population: a systematic review. Int J Clin Pract 2011;65:1180-92.

39. Scott LJ. Enzalutamide: A Review in Castration-Resistant Prostate Cancer. Drugs 2018;78:1913-24.

40. Hussain M, Fizazi K, Saad F, et al. Enzalutamide in Men with Nonmetastatic, Castration-Resistant Prostate Cancer. N Engl J Med 2018;378:2465-74.

41. Scher HI, Fizazi K, Saad F, et al. Increased survival with enzalutamide in prostate cancer after chemotherapy. $\mathrm{N}$ Engl J Med 2012;367:1187-97.

42. Antonarakis ES, Lu C, Wang H, et al. AR-V7 and resistance to enzalutamide and abiraterone in prostate cancer. N Engl J Med 2014;371:1028-38.

43. Joseph JD, Lu N, Qian J, et al. A clinically relevant androgen receptor mutation confers resistance to secondgeneration antiandrogens enzalutamide and ARN-509. Cancer Discov 2013;3:1020-9.

44. Korpal M, Korn JM, Gao X, et al. An F876L mutation in androgen receptor confers genetic and phenotypic resistance to MDV3100 (enzalutamide). Cancer Discov 2013;3:1030-43.

45. Gustafson JL, Neklesa TK, Cox CS, et al. SmallMolecule-Mediated Degradation of the Androgen Receptor through Hydrophobic Tagging. Angew Chem Int Ed Engl 2015;54:9659-62.

46. Salami J, Alabi S, Willard RR, et al. Androgen receptor degradation by the proteolysis-targeting chimera ARCC4 outperforms enzalutamide in cellular models of prostate cancer drug resistance. Commun Biol 2018;1:100.

47. Han X, Wang C, Qin C, et al. Discovery of ARD-69 as a Highly Potent Proteolysis Targeting Chimera (PROTAC) Degrader of Androgen Receptor (AR) for the Treatment of Prostate Cancer. J Med Chem 2019;62:941-64. 
48. Kregel S, Wang C, Han X, et al. Androgen receptor degraders overcome common resistance mechanisms developed during prostate cancer treatment. Neoplasia 2020;22:111-9.

49. Zhao L, Han X, Lu J, et al. A highly potent PROTAC androgen receptor (AR) degrader ARD-61 effectively inhibits AR-positive breast cancer cell growth in vitro and tumor growth in vivo. Neoplasia 2020;22:522-32.

50. Han X, Zhao L, Xiang W, et al. Discovery of Highly Potent and Efficient PROTAC Degraders of Androgen Receptor (AR) by Employing Weak Binding Affinity VHL E3 Ligase Ligands. J Med Chem 2019;62:11218-31.

51. Malumbres M. Cyclin-dependent kinases. Genome Biol 2014;15:122.

52. Asghar U, Witkiewicz AK, Turner NC, et al. The history and future of targeting cyclin-dependent kinases in cancer therapy. Nat Rev Drug Discov 2015;14:130-46.

53. Kase AM, Copland Iii JA, Tan W. Novel Therapeutic Strategies for CDK4/6 Inhibitors in Metastatic Castrate-Resistant Prostate Cancer. Onco Targets Ther

Cite this article as: Chen X, Shen H, Shao Y, Ma Q, Niu Y, Shang Z. A narrative review of proteolytic targeting chimeras (PROTACs): future perspective for prostate cancer therapy. Transl Androl Urol 2021;10(2):954-962. doi: 10.21037/tau-201357
2020;13:10499-513.

54. Laroche-Clary A, Chaire V, Algeo MP, et al. Combined targeting of MDM2 and CDK4 is synergistic in dedifferentiated liposarcomas. J Hematol Oncol 2017;10:123.

55. Steinebach C, Ng YLD, Sosič I, et al. Systematic exploration of different E3 ubiquitin ligases: an approach towards potent and selective CDK6 degraders. Chem Sci 2020;11:3474-86.

56. Xie H, Liang JJ, Wang YL, et al. The design, synthesis and anti-tumor mechanism study of new androgen receptor degrader. Eur J Med Chem 2020;204:112512.

57. Takwale AD, Jo SH, Jeon YU, et al. Design and characterization of cereblon-mediated androgen receptor proteolysis-targeting chimeras. Eur J Med Chem 2020;208:112769.

58. Davies AH, Beltran H, Zoubeidi A. Cellular plasticity and the neuroendocrine phenotype in prostate cancer. Nat Rev Urol 2018;15:271-86. 